\title{
Syntheses, structures, and photoluminescence of 1-D lanthanide coordination polymers $\dagger$
}

\author{
Xiaoping Yang, ${ }^{a}$ Richard A. Jones, ${ }^{* a}$ Joseph H. Rivers ${ }^{a}$ and Wai-Kwok Wong $* b$ \\ Received 29th May 2009, Accepted 15th October 2009 \\ First published as an Advance Article on the web 29th October 2009 \\ DOI: 10.1039/b910588c
}

Five new lanthanide 1-D coordination polymers are reported which are formed from flexible salen type Schiff-base ligands $\mathrm{H}_{2} \mathrm{~L}$ and $\mathrm{H}_{2} \mathrm{~L}^{\prime}\left(\mathrm{H}_{2} \mathrm{~L}=N\right.$, $\mathrm{N}^{1}$-ethylene bis(salicylideneimine); $\mathrm{H}_{2} \mathrm{~L}^{\prime}=N, \mathrm{~N}^{1}$-bis(3methoxysalicylidene)ethylene-1,2-diamine). The polymeric structures are formed by bridging neutral $\mathrm{H}_{2} \mathrm{~L}$ units in the case of $\left\{\left[\mathrm{Ln}_{2} \mathrm{~L}_{2}\left(\mathrm{CF}_{3} \mathrm{SO}_{3}\right)\left(\mathrm{H}_{2} \mathrm{~L}\right)_{4}(\mathrm{MeOH})\right] \cdot \mathrm{CF}_{3} \mathrm{SO}_{3}\right\}_{\mathrm{n}}(\mathrm{Ln}=\mathrm{Eu}(\mathbf{1}), \mathrm{Nd}(2)$ and $\mathrm{Er}(3))$, and by acetate $\left(\mathrm{OAc}^{-}\right)$groups in $\left[\mathrm{Yb}_{2}(\mathrm{~L})_{2}(\mathrm{OAc})_{2}(\mathrm{MeOH})_{2}\right]_{\mathrm{n}}(\mathbf{4})$ and $\left\{\left[\mathrm{Tb}_{3}\left(\mathrm{~L}^{\prime}\right)_{2}(\mathrm{OAc})_{5}\right] \cdot \mathrm{Et}_{2} \mathrm{O}\right.$.

$\left.(\mathrm{MeOH})_{0.5}\right\}_{\mathrm{n}}(\mathbf{5})$. The structures of $\mathbf{1}-\mathbf{5}$ were determined by single crystal X-ray crystallographic studies and the luminescence properties of $\mathbf{1}$ and $\mathbf{5} \mathrm{in} \mathrm{MeOH}$ solution were determined.

\section{Introduction}

There is currently considerable interest in the rational design and synthesis of coordination framework materials prepared from multifunctional organic ligands and lanthanide ions since they have potential applications related to luminescence, magnetism, gas absorption and sensing. ${ }^{1}$ Control over the lanthanide coordination environment, and therefore a coordination framework is challenging due to high coordination numbers and flexible coordination geometries that are encountered in Ln(III) chemistry. ${ }^{2}$ In addition, the structures and compositions of lanthanide frameworks are often significantly influenced not only by ligand structures but also by counterions and reaction conditions. ${ }^{1,3}$ The majority of $\mathrm{Ln}$ (III) coordination polymers reported so far have employed polydentate rigid ligands, such as multicarboxylic acids, pyridine $N$-oxide-based ligands, carboxyphenyl porphyrin and various other macrocyclic ligands. ${ }^{4}$ It is interesting to note that while more flexible ligands featuring $\mathrm{S}, \mathrm{N}$ or $\mathrm{O}$ atom donors have been employed in the construction of d-block transition metal frameworks relatively few studies involving more flexible linkers with lanthanides have been reported. ${ }^{5}$

Schiff-base ligands are among the most widely studied chelators for metal ions owing to their relatively easy synthesis and their versatility in the formation of stable complexes. Our research has recently focused on the use of multidentate Schiff-base ligands for the preparation of new luminescent lanthanide complexes. ${ }^{6}$ Although the first X-ray crystal structure of a lanthanide coordination polymer from the reaction of the neutral ligand $\mathrm{H}_{2} \mathrm{~L}$ (Scheme 1) with $\mathrm{La}\left(\mathrm{NO}_{3}\right)_{3}$ hydrate was reported by Xie in $1999^{7}$ there are relatively few reports of lanthanide coordination polymers which employ salen type Schiff-base ligands. ${ }^{8}$ We recently reported that under

${ }^{a}$ Department of Chemistry and Biochemistry, The University of Texas at Austin, 1 University Station, A5300, Austin, 78712-0165, TX, USA. E-mail: rajones@mail.utexas.edu

${ }^{b}$ Department of Chemistry and Centre for Advanced Luminescence Materials, Hong Kong Baptist University, Waterloo Road, Kowloon Tong, Hong Kong, China. E-mail:wkwong@hkbu.edu.hk

$\dagger$ Electronic supplementary information (ESI) available: ESI-MS data for 2. CCDC reference numbers 733181-733185. For ESI and crystallographic data in CIF or other electronic format see DOI: 10.1039/b910588c

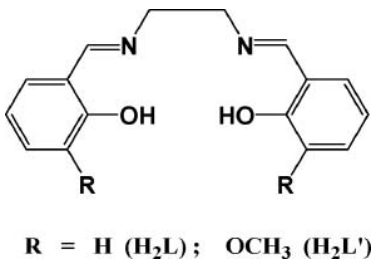

Scheme 1 Schiff-base ligands $\mathrm{H}_{2} \mathrm{~L}$ and $\mathrm{H}_{2} \mathrm{~L}^{\prime}$.

neutral conditions the products from reactions of $\mathrm{LnX}_{3} \cdot \mathrm{nH}_{2} \mathrm{O}$ ( $\mathrm{X}=\mathrm{OAc}^{-}, \mathrm{NO}_{3}{ }^{-}$and $\mathrm{Cl}^{-}$) with $\mathrm{H}_{2} \mathrm{~L}$ (salen) were highly dependant on the nature of the anion $\mathrm{X}^{-}$. When $\mathrm{X}=$ acetate $\left(\mathrm{OAc}^{-}\right)$the neutral binary species $\mathrm{Tb}_{4} \mathrm{~L}_{6}$ was formed. ${ }^{6 a}$ However, when $\mathrm{X}=\mathrm{Cl}^{-}$ or $\mathrm{NO}_{3}{ }^{-}$simple dinuclear complexes of formula $(\mathrm{XLnL})_{2}\left(\mu-\mathrm{H}_{2} \mathrm{~L}\right)$ are formed in which a neutral salen molecule $\left(\mathrm{H}_{2} \mathrm{~L}\right)$ acts a simple bridge between two metal centers. In this report we describe further examples of $\mathrm{Ln}$ (III) 1-D coordination polymers. These materials fall into two categories. Firstly, complexes in which a neutral $\mathrm{H}_{2} \mathrm{~L}$ salen group bridges between metal centers through phenolic$\mathrm{OH}$ groups; $\left\{\left[\mathrm{Ln}_{2} \mathrm{~L}_{2}\left(\mathrm{CF}_{3} \mathrm{SO}_{3}\right)\left(\mathrm{H}_{2} \mathrm{~L}\right)_{4}(\mathrm{MeOH})\left(\mathrm{H}_{2} O\right)\right] \cdot \mathrm{CF}_{3} \mathrm{SO}_{3}\right\}_{\mathrm{n}}$ $(\mathrm{Ln}=\mathrm{Eu}(1), \mathrm{Nd}$ (2) and $\operatorname{Er}(3))$. Secondly, complexes in which 1-D coordination polymers are created by the use of acetate $\left(\mathrm{OAc}^{-}\right)$groups which bridge between both bimetallic and trimetallic $\mathrm{Ln}(\mathrm{III})$ clusters $\left[\mathrm{Yb}_{2}(\mathrm{~L})_{2}(\mathrm{OAc})_{2}(\mathrm{MeOH})_{2}\right]_{\mathrm{n}}(4)$ and $\left\{\left[\mathrm{Tb}_{3}\left(\mathrm{~L}^{\prime}\right)_{2}(\mathrm{OAc})_{5}\right] \cdot \mathrm{Et}_{2} \mathrm{O} \cdot(\mathrm{MeOH})_{0.5}\right\}_{\mathrm{n}}$ (5). Interestingly the reaction conditions used for the synthesis of these materials are critical and involve the use of $\mathrm{Et}_{3} \mathrm{~N}$ in refluxing $\mathrm{MeOH}$. The use of stronger bases often result in the formation of $\mathrm{Ln}(\mathrm{OH})$ species.

\section{Experimental section}

\section{General considerations}

All reactions were performed under dry oxygen-free dinitrogen atmospheres using standard Schlenk techniques. The Schiff-base ligands tend to be slightly unstable with respect to rearrangements or hydrolysis under the reaction conditions, especially in the presence of air. The reactions are therefore conducted under an inert atmosphere. After coordination to lanthanide ions the Schiff-base ligand is far more stable in air, especially at room 
temperature. The metal complexes can therefore be crystallized in air at this stage of the procedure. Metal salts, triethylamine and other solvents were purchased from Aldrich and used directly without further purification. Schiff-base ligands $\mathrm{H}_{2} \mathrm{~L}$ and $\mathrm{H}_{2} \mathrm{~L}^{\prime}$ were prepared according to well-established procedures. ${ }^{9}$ Physical measurements: IR: Nicolet IR 200 FTIR spectrometer. Melting points were obtained in sealed glass capillaries under dinitrogen and are uncorrected. Satisfactory microanalytical data $(\mathrm{C}, \mathrm{H}$, $N$ ) was obtained for all compounds. Absorption spectra were obtained on a BECKMAN DU 640 spectrophotometer, excitation and visible emission spectra on a QuantaMaster PTI fluorimeter.

\section{Synthesis of complexes 1-5}

$\left\{\left[\mathrm{Eu}_{2} \mathrm{~L}_{2}\left(\mathrm{CF}_{3} \mathrm{SO}_{3}\right)\left(\mathrm{H}_{2} \mathbf{L}\right)_{4}(\mathrm{MeOH})\right] \cdot \mathrm{CF}_{3} \mathrm{SO}_{3}\right\}_{\mathrm{n}} \quad$ (1). Triethylamine $(2 \mathrm{~mL}$ of a $0.1 \mathrm{M} \mathrm{MeCN}$ solution) was added to a solution of $\mathrm{H}_{2} \mathrm{~L}(0.027 \mathrm{~g}, 0.10 \mathrm{mmol})$ and $\mathrm{Eu}\left(\mathrm{CF}_{3} \mathrm{SO}_{3}\right)_{3}(0.060 \mathrm{~g}$, $0.10 \mathrm{mmol})$ in $\mathrm{MeOH}(25 \mathrm{ml})$. The resulting solution was stirred and heated under reflux for one day. The solution was then cooled to room temperature and filtered. The filtrate was allowed to evaporate in the air. Yellow single crystals of $\mathbf{1}$ were formed after two weeks. Yield (base on $\mathrm{H}_{2} \mathrm{~L}$ ): $0.011 \mathrm{~g}(30 \%)$. m. p. > $300{ }^{\circ} \mathrm{C}$ (dec.). ESI-MS (MeOH) m/z: $955\left(\left[\mathrm{Eu}_{2} \mathrm{~L}_{2}\left(\mathrm{H}_{2} \mathrm{~L}\right)_{4}\right]^{2+}\right)$. IR $\left(\mathrm{CH}_{3} \mathrm{OH}, \mathrm{cm}^{-1}\right)$ : 3464(s), $2998(\mathrm{~m}), 2949(\mathrm{~m}), 2835$ (m), 1634 (s), 1536 (w), 1470 (m), 1442 (m), 1397 (w), 1343 (w), 1278 (s), 1254 (s), $1225(\mathrm{~m}), 1172(\mathrm{~m}), 1111(\mathrm{w}), 11086(\mathrm{w}), 1029(\mathrm{~s}), 759(\mathrm{~m})$, $641(\mathrm{~m})$.

$\left\{\left[\mathbf{N d}_{2} \mathbf{L}_{2}\left(\mathbf{C F}_{3} \mathbf{S O}_{3}\right)\left(\mathbf{H}_{2} \mathbf{L}\right)_{4}(\mathbf{M e O H}) \cdot \cdot \mathbf{C F}_{3} \mathbf{S O}_{3}\right\}_{n}\right.$ (2). The procedure was the same as that for 1 using $\mathrm{Nd}\left(\mathrm{CF}_{3} \mathrm{SO}_{3}\right)_{3}$. Yellow single crystals of $\mathbf{2}$ were formed after two weeks. Yield (base on $\left.\mathrm{H}_{2} \mathrm{~L}\right): 0.012 \mathrm{~g}(33 \%)$. m. p. $>300{ }^{\circ} \mathrm{C}$ (dec.). ESI-MS (MeOH) $m / z$ : $947\left(\left[\mathrm{Nd}_{2} \mathrm{~L}_{2}\left(\mathrm{H}_{2} \mathrm{~L}\right)_{4}\right]^{2+}\right), 1088\left(\left[\mathrm{Nd}_{2} \mathrm{~L}_{2}(\mathrm{HL})\right]^{+}\right), 1237$ $\left(\left[\mathrm{Nd}_{2} \mathrm{~L}_{2}\left(\mathrm{H}_{2} \mathrm{~L}\right)\left(\mathrm{CF}_{3} \mathrm{SO}_{3}\right)\right]^{+}\right) . \mathrm{IR}\left(\mathrm{CH}_{3} \mathrm{OH}, \mathrm{cm}^{-1}\right): 3358(\mathrm{~m}), 3174(\mathrm{~m})$, 3011 (m), 1629 (s), 1535 (w), 1470 (m), 1442 (m), 1401 (w), 1339 (w), $1274(\mathrm{~s}), 1245(\mathrm{~s}), 1170(\mathrm{~s}), 1029$ (s), $755(\mathrm{~m}), 632(\mathrm{~m})$.

$\left\{\left[\mathbf{E r}_{2} \mathbf{L}_{2}\left(\mathbf{C F}_{3} \mathbf{S O}_{3}\right)\left(\mathbf{H}_{2} \mathbf{L}\right)_{4}(\mathbf{M e O H})\right] \cdot \mathbf{C F}_{3} \mathbf{S O}_{3}\right\}_{n}$ (3). The procedure was the same as that for 1 using $\operatorname{Er}\left(\mathrm{CF}_{3} \mathrm{SO}_{3}\right)_{3}$. Yellow single crystals of $\mathbf{3}$ were formed after two weeks. Yield (base on $\left.\mathrm{H}_{2} \mathrm{~L}\right): 0.015 \mathrm{~g}(40 \%)$. m. p. $>306{ }^{\circ} \mathrm{C}(\mathrm{dec})$. $\left(\left[\mathrm{Er}_{2} \mathrm{~L}_{2}\left(\mathrm{H}_{2} \mathrm{~L}\right)\left(\mathrm{CF}_{3} \mathrm{SO}_{3}\right)\right]^{+}\right)$. IR $\left(\mathrm{CH}_{3} \mathrm{OH}, \mathrm{cm}^{-1}\right): 3312(\mathrm{~m}), 3008(\mathrm{~m})$, 1631 (s), 1539 (w), 1472 (m), 1445 (m), 1396 (w), 1341 (w), 1276 (s), 1251 (s), $1168(\mathrm{~s}), 1017$ (s), $756(\mathrm{~m}), 630(\mathrm{~m})$.

$\left[\mathbf{Y b}_{2} \mathbf{L}_{2}(\mathbf{O A c})_{2}(\mathbf{M e O H})_{2}\right]_{\mathbf{n}}(\mathbf{4})$. The procedure was the same as that for 1 using $\mathrm{Yb}(\mathrm{OAc})_{3} \cdot 4 \mathrm{H}_{2} \mathrm{O}(0.035 \mathrm{~g}, 0.10 \mathrm{mmol})$. Yellow single crystals of 3 were formed after two weeks. Yield $0.058 \mathrm{~g}$ $(55 \%)$. m. p. $>295{ }^{\circ} \mathrm{C}$ (dec.). ESI-MS (MeOH) $\mathrm{m} / z: 1002$ $\left(\left[\mathrm{Yb}_{2} \mathrm{~L}_{2}(\mathrm{OAc})(\mathrm{MeOH})_{2}\right]^{+}\right)$. IR $\left(\mathrm{CH}_{3} \mathrm{OH}, \mathrm{cm}^{-1}\right): 3411(\mathrm{~s}), 1634(\mathrm{~s})$, 1548 (s), $1454(\mathrm{~m}), 1409$ (m), 1335 (w), $1286(\mathrm{w}), 1237$ (w), 1217 (w), $1074(\mathrm{w}), 951(\mathrm{w}), 739(\mathrm{~m}), 677(\mathrm{~m})$.

$\left\{\left[\mathbf{T b}_{3}\left(\mathbf{L}^{\prime}\right)_{2}(\mathbf{O A c})_{5}\right] \cdot \mathbf{E t}_{2} \mathbf{O} \cdot(\mathbf{M e O H})_{0.5}\right\}_{\mathrm{n}}$

(5). Triethylamine ( $2 \mathrm{~mL}$ of a $0.1 \mathrm{M} \mathrm{MeCN}$ solution) was added to a solution of $\mathrm{H}_{2} \mathrm{~L}^{\prime}(0.033 \mathrm{~g}, 0.10 \mathrm{mmol})$ and $\mathrm{Tb}(\mathrm{OAc})_{3} \cdot 4 \mathrm{H}_{2} \mathrm{O}(0.034 \mathrm{~g}$, $0.10 \mathrm{mmol})$ in $\mathrm{MeOH}(20 \mathrm{ml})$. The resulting solution was stirred and heated under reflux for one day. The solution was then cooled to room temperature and filtered. Diethylether was allowed to diffuse slowly into this solution at room temperature and yellow single crystals were formed in three weeks. Yield (base on
$\left.\mathrm{Tb}(\mathrm{OAc})_{3} \cdot 4 \mathrm{H}_{2} \mathrm{O}\right): 0.025 \mathrm{~g}(50 \%)$. m. p. $>315^{\circ} \mathrm{C}(\mathrm{dec}$.). ESI-MS (MeOH) $m / z: 1425\left(\left[\mathrm{~Tb}_{3} \mathrm{~L}^{\prime}\left(\mathrm{HL}^{\prime}\right)(\mathrm{OAc})_{5}\right]^{+}\right)$. IR $\left(\mathrm{CH}_{3} \mathrm{OH}, \mathrm{cm}^{-1}\right)$ : 3101 (s), $2884(\mathrm{~m}), 1638$ (s), 1548 (s), 1454 (m), 1417 (m), 1342 (w), $1221(\mathrm{~m}), 1168(\mathrm{w}), 1082(\mathrm{w}), 951(\mathrm{w}), 678(\mathrm{~m})$.

\section{X-Ray Crystallography}

Data were collected on a Nonius Kappa CCD diffractometer with graphite monochromated Mo-K $\alpha$ radiation $(\lambda=0.71073 \AA)$ at $153 \mathrm{~K}$. Absorption corrections were applied using GAUSSIAN. The structures were solved by direct methods and refined anisotropically using full-matrix least-squares methods with the SHELX 97 program package. ${ }^{10}$ Coordinates of the non-hydrogen atoms were refined anisotropically, while hydrogen atoms were included in the calculation isotropically but not refined. Neutral atom scattering factors were taken from Cromer and Waber. ${ }^{11}$

\section{Results and discussion}

In the presence of the weak base $\mathrm{Et}_{3} \mathrm{~N}$, reactions of Schiff-base ligands $\mathrm{H}_{2} \mathrm{~L}$ and $\mathrm{H}_{2} \mathrm{~L}^{\prime}$ with $\mathrm{Ln}\left(\mathrm{CF}_{3} \mathrm{SO}_{3}\right)_{3}(\mathrm{Ln}=\mathrm{Eu}, \mathrm{Nd}$ and $\mathrm{Er}), \mathrm{Yb}(\mathrm{OAc})_{3} \cdot 4 \mathrm{H}_{2} \mathrm{O}$ and $\mathrm{Tb}(\mathrm{OAc})_{3} \cdot 4 \mathrm{H}_{2} \mathrm{O}$ in refluxing $\mathrm{MeOH}$ produced yellow solutions from which compounds 1-5 may be isolated as yellow crystalline solids in $30-50 \%$ yields. The solid state structures of 1-5 were determined by single crystal X-ray crystallographic studies. Crystallographic data for all polymers are presented in Table 1 and selected bond lengths and angles are given in Table 2-6.

\section{Neutral Salen Bridged 1-D Polymers}

Complexes 1-3 are isomorphous and have the general formula $\left\{\left[\mathrm{Ln}_{2} \mathrm{~L}_{2}\left(\mathrm{CF}_{3} \mathrm{SO}_{3}\right)\left(\mathrm{H}_{2} \mathrm{~L}\right)_{4}(\mathrm{MeOH})\right] \cdot \mathrm{CF}_{3} \mathrm{SO}_{3}\right\}_{\mathrm{n}}(\mathrm{Ln}=\mathrm{Eu}(\mathbf{1}), \mathrm{Nd}(\mathbf{2})$ and $\operatorname{Er}(3))$. The compounds crystallize in the triclinic space group $P 1$ with two $\mathrm{Ln}^{3+}$ ions, two $\mathrm{L}^{2-}$ ligands, four neutral $\mathrm{H}_{2} \mathrm{~L}$ groups, two $\mathrm{CF}_{3} \mathrm{SO}_{3}{ }^{-}$anions and one coordinated $\mathrm{MeOH}$ molecule per asymmetric unit. A view of the central $\mathrm{Eu}_{2}$ structure of $\mathbf{1}$ is shown in Fig. 1. Each $\mathrm{Eu}(1)$ ion is coordinated to a deprotonated salen group $\left(\mathrm{L}^{2-}\right)$ and two neutral salen ligands $\left(\mathrm{H}_{2} \mathrm{~L}\right)$ while $\mathrm{Eu}(2)$ is coordinated to three salen ligands and one $\mathrm{L}^{2-} . \mathrm{Eu}(1)$ and $\mathrm{Eu}(2)$ are bridged by a neutral salen ligand with a separation of $10.171 \AA$, forming a 1-D polymeric structure. The central $\mathrm{O}_{2} \mathrm{~N}_{2}$ binding pocket of each $\mathrm{L}^{2-}$ group is in an approximately planar arrangement. The non bridging, neutral $\mathrm{H}_{2} \mathrm{~L}$ groups bind via one phenolic $\mathrm{OH}$ group leaving the other $\mathrm{OH}$ group of the ligand not bound to a metal. Both metal centers have coordination numbers of 8. $\mathrm{Eu}(1)$ also bears a coordinated $\mathrm{CF}_{3} \mathrm{SO}_{3}{ }^{-}$group via an oxygen atom while $\mathrm{Eu}(2)$ bears a single coordinated $\mathrm{MeOH}$ molecule. In complexes 1-3, the average distance for the $\mathrm{Ln}-\mathrm{O}$ (phenolic) interactions (2.380 $\mathrm{A} \mathbf{1}, 2.448 \AA 2,2.319 \AA 3)$ and Ln-N (2.611 1, $2.682 \AA 2,2.548 \AA 3$ ) are comparable to those found in the literature. ${ }^{6,12}$ The Ln-Ln distances in $\mathbf{2}(10.210 \AA)$ and $\mathbf{3}(10.041 \AA)$ are similar to that observed in $\mathbf{1}$. A view of the 1-D polymeric chain-like structure of $\mathbf{1}$ is shown in Fig. 2. The 1-D polymeric structure is formed by a zig-zag chain of alternating $\mathrm{Eu}^{3+}$ ions and $\mathrm{H}_{2} \mathrm{~L}$ (salen) ligands which bridge as neutral ligands between the metal centers (Scheme 2, type II). 
Table 1 Crystal data and structure refinement for compounds 1-5

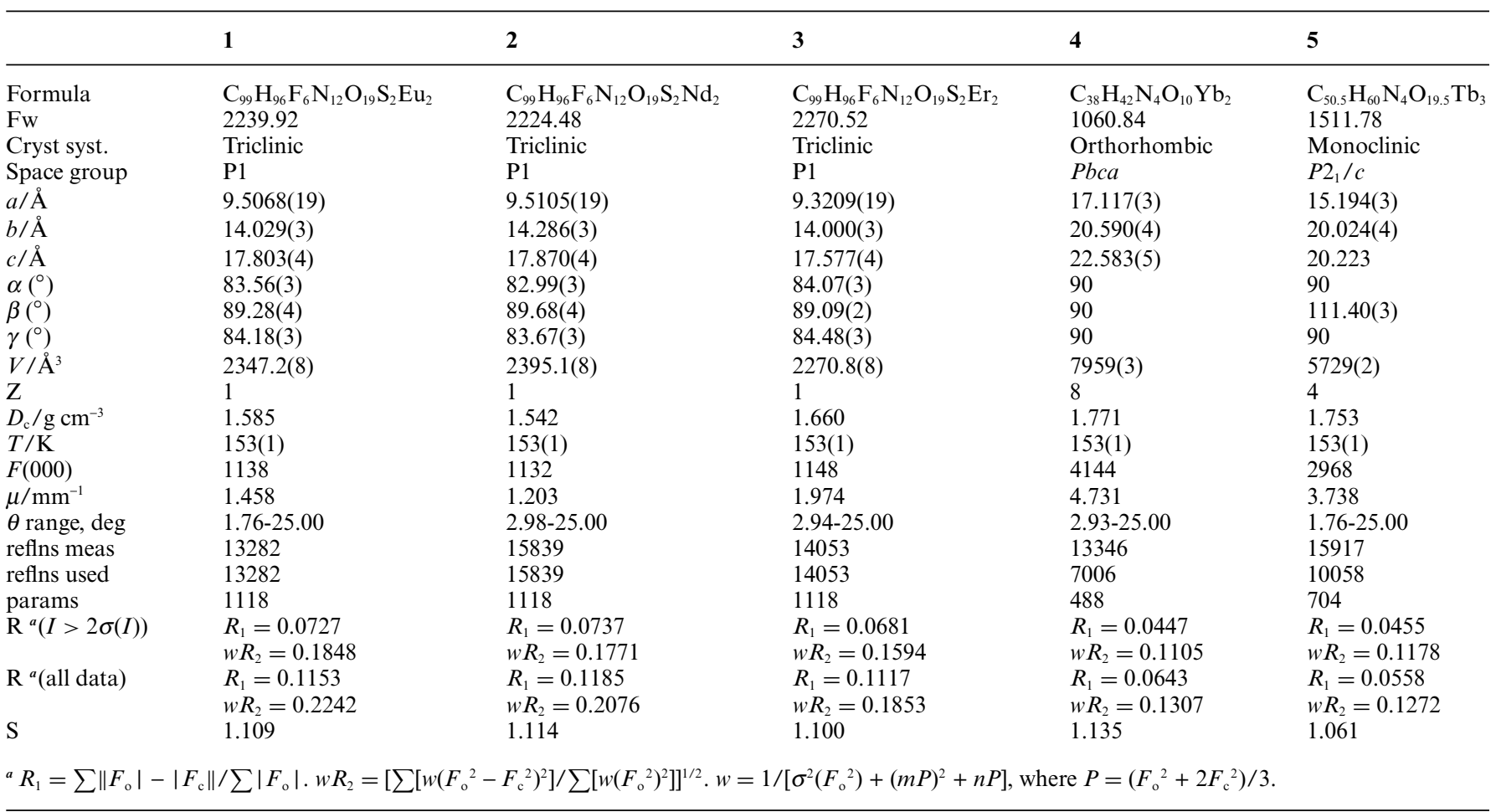

Table 2 Selected bond lengths $(\AA)$ and angles $\left({ }^{\circ}\right)$ for 1. Symmetry operator $(1+x, y, 1+z)$ generates equivalent atoms marked with "\#"

\begin{tabular}{llll}
\hline $\mathrm{Eu}(1)-\mathrm{O}(2 \mathrm{~B})$ & $2.30(2)$ & $\mathrm{Eu}(1)-\mathrm{O}(1 \mathrm{~B})$ & $2.377(16)$ \\
$\mathrm{Eu}(1)-\mathrm{O}(2 E) \# 1$ & $2.392(16)$ & $\mathrm{Eu}(1)-\mathrm{O}(1)$ & $2.404(18)$ \\
$\mathrm{Eu}(1)-\mathrm{O}(1 \mathrm{C})$ & $2.431(18)$ & $\mathrm{Eu}(1)-\mathrm{O}(3)$ & $2.482(8)$ \\
$\mathrm{Eu}(1)-\mathrm{N}(1 \mathrm{~B})$ & $2.58(2)$ & $\mathrm{Eu}(1)-\mathrm{N}(2 \mathrm{~B})$ & $2.70(2)$ \\
$\mathrm{Eu}(2)-\mathrm{O}(2 \mathrm{D})$ & $2.325(18)$ & $\mathrm{Eu}(2)-\mathrm{O}(1 E)$ & $2.348(18)$ \\
$\mathrm{Eu}(2)-\mathrm{O}(2 \mathrm{C})$ & $2.380(19)$ & $\mathrm{Eu}(2)-\mathrm{O}(1 \mathrm{D})$ & $2.400(17)$ \\
$\mathrm{Eu}(2)-\mathrm{O}(2 \mathrm{~F})$ & $2.439(19)$ & $\mathrm{Eu}(2)-\mathrm{N}(2 \mathrm{D})$ & $2.59(3)$ \\
$\mathrm{Eu}(2)-\mathrm{O}(9)$ & $2.561(14)$ & $\mathrm{Eu}(2)-\mathrm{N}(1 \mathrm{D})$ & $2.59(2)$ \\
$\mathrm{O}(2 \mathrm{~B})-\mathrm{Eu}(1)-\mathrm{O}(1 \mathrm{~B})$ & $156.1(6)$ & $\mathrm{O}(2 \mathrm{~B})-\mathrm{Eu}(1)-\mathrm{O}(2 E) \# 1$ & $95.2(7)$ \\
$\mathrm{O}(1 \mathrm{~B})-\mathrm{Eu}(1)-\mathrm{O}(2 E) \# 1$ & $71.5(6)$ & $\mathrm{O}(2 \mathrm{~B})-\mathrm{Eu}(1)-\mathrm{O}(1)$ & $83.9(7)$ \\
$\mathrm{O}(1 \mathrm{~B})-\mathrm{Eu}(1)-\mathrm{O}(1)$ & $109.6(6)$ & $\mathrm{O}(2 E) \# 1-\mathrm{Eu}(1)-\mathrm{O}(1)$ & $72.3(6)$ \\
$\mathrm{O}(2 \mathrm{~B})-\mathrm{Eu}(1)-\mathrm{O}(1 \mathrm{C})$ & $74.4(7)$ & $\mathrm{O}(1 \mathrm{~B})-\mathrm{Eu}(1)-\mathrm{O}(1 \mathrm{C})$ & $83.5(6)$ \\
$\mathrm{O}(2 E) \# 1-\mathrm{Eu}(1)-\mathrm{O}(1 \mathrm{C})$ & $79.2(6)$ & $\mathrm{O}(1)-\mathrm{Eu}(1)-\mathrm{O}(1 \mathrm{C})$ & $142.2(7)$ \\
$\mathrm{O}(2 \mathrm{~B})-\mathrm{Eu}(1)-\mathrm{N}(1 \mathrm{~B})$ & $133.9(7)$ & $\mathrm{O}(1 \mathrm{~B})-\mathrm{Eu}(1)-\mathrm{N}(1 \mathrm{~B})$ & $69.3(6)$ \\
$\mathrm{O}(2 E) \# 1-\mathrm{Eu}(1)-\mathrm{N}(1 \mathrm{~B})$ & $119.8(6)$ & $\mathrm{O}(1)-\mathrm{Eu}(1)-\mathrm{N}(1 \mathrm{~B})$ & $80.1(6)$ \\
$\mathrm{O}(1 \mathrm{C})-\mathrm{Eu}(1)-\mathrm{N}(1 \mathrm{~B})$ & $136.8(6)$ & $\mathrm{O}(2 \mathrm{~B})-\mathrm{Eu}(1)-\mathrm{N}(2 \mathrm{~B})$ & $68.2(7)$ \\
$\mathrm{O}(1 \mathrm{~B})-\mathrm{Eu}(1)-\mathrm{N}(2 \mathrm{~B})$ & $134.2(7)$ & $\mathrm{O}(2 E) \# 1-\mathrm{Eu}(1)-\mathrm{N}(2 \mathrm{~B})$ & $141.2(6)$ \\
$\mathrm{O}(1)-\mathrm{Eu}(1)-\mathrm{N}(2 \mathrm{~B})$ & $71.3(6)$ & $\mathrm{O}(1 \mathrm{C})-\mathrm{Eu}(1)-\mathrm{N}(2 \mathrm{~B})$ & $125.0(6)$ \\
$\mathrm{N}(1 \mathrm{~B})-\mathrm{Eu}(1)-\mathrm{N}(2 \mathrm{~B})$ & $65.8(7)$ & & \\
\hline
\end{tabular}

\section{1-D Polymers with Acetate $\left(\mathrm{OAc}^{-}\right)$Bridges}

$\mathbf{Y b}_{2}$. The reaction of $\mathrm{Yb}(\mathrm{OAc})_{3} \cdot 4 \mathrm{H}_{2} \mathrm{O}$ with salen in the presence of $\mathrm{Et}_{3} \mathrm{~N}$ in refluxing $\mathrm{MeOH}$ produces the 1-D polymeric material $\left[\mathrm{Yb}_{2}(\mathrm{~L})_{2}(\mathrm{OAc})_{2}(\mathrm{MeOH})_{2}\right]_{\mathrm{n}}$ (4) in $55 \%$ yield. The compound crystallizes in the orthorhombic space group $\mathrm{Pbca}$ with two unique $\mathrm{Yb}$ atoms, two $\mathrm{L}^{2-}$ anions, two $\mathrm{OAc}^{-}$groups and two coordinated $\mathrm{MeOH}$ molecules per asymmetric unit. A view of the central $\mathrm{Yb}_{2}$ unit of $\mathbf{4}$ is shown in Fig. 3. Each $\mathrm{Yb}^{3+}$ ion is bound by the $\mathrm{N}_{2} \mathrm{O}_{2}$ cavity of a deprotonated salen ligand $\left(\mathrm{L}^{2-}\right)$ and is 8-coordinate. $\mathrm{Yb}(1)$ ion has two coordinated $\mathrm{MeOH}$ molecules and is linked to $\mathrm{Yb}(2)$. The shorter distance $\mathrm{Yb}(1)-\mathrm{Yb}(2)$ is $3.948 \AA$. The longer distance between these two ions, bridged
Table 3 Selected bond lengths $(\AA)$ and angles $\left({ }^{\circ}\right)$ for 2 . Symmetry operator $(1+x, y, 1+z)$ generates equivalent atoms marked with "\#"

\begin{tabular}{llll}
\hline $\mathrm{Nd}(1)-\mathrm{O}(2 \mathrm{~B})$ & $2.387(17)$ & $\mathrm{Nd}(1)-\mathrm{O}(2 E) \# 1$ & $2.429(16)$ \\
$\mathrm{Nd}(1)-\mathrm{O}(1 \mathrm{C})$ & $2.436(15)$ & $\mathrm{Nd}(1)-\mathrm{O}(1 \mathrm{~B})$ & $2.439(15)$ \\
$\mathrm{Nd}(1)-\mathrm{O}(1)$ & $2.513(17)$ & $\mathrm{Nd}(1)-\mathrm{O}(3)$ & $2.544(7)$ \\
$\mathrm{Nd}(1)-\mathrm{N}(1 \mathrm{~B})$ & $2.668(19)$ & $\mathrm{Nd}(1)-\mathrm{N}(2 \mathrm{~B})$ & $2.694(18)$ \\
$\mathrm{Nd}(2)-\mathrm{O}(2 \mathrm{D})$ & $2.409(17)$ & $\mathrm{Nd}(2)-\mathrm{O}(1 E)$ & $2.435(13)$ \\
$\mathrm{Nd}(2)-\mathrm{O}(1 \mathrm{D})$ & $2.456(15)$ & $\mathrm{Nd}(2)-\mathrm{O}(2 \mathrm{~F})$ & $2.473(14)$ \\
$\mathrm{Nd}(2)-\mathrm{O}(2 \mathrm{C})$ & $2.498(16)$ & $\mathrm{Nd}(2)-\mathrm{O}(9)$ & $2.636(11)$ \\
$\mathrm{Nd}(2)-\mathrm{N}(2 \mathrm{D})$ & $2.637(19)$ & $\mathrm{Nd}(2)-\mathrm{N}(1 \mathrm{D})$ & $2.74(2)$ \\
$\mathrm{O}(2 \mathrm{~B})-\mathrm{Nd}(1)-\mathrm{O}(2 E) \# 1$ & $98.3(6)$ & $\mathrm{O}(2 \mathrm{~B})-\mathrm{Nd}(1)-\mathrm{O}(1 \mathrm{C})$ & $73.9(6)$ \\
$\mathrm{O}(2 E) \# 1-\mathrm{Nd}(1)-\mathrm{O}(1 \mathrm{C})$ & $80.6(5)$ & $\mathrm{O}(2 \mathrm{~B})-\mathrm{Nd}(1)-\mathrm{O}(1 \mathrm{~B})$ & $159.2(6)$ \\
$\mathrm{O}(2 E) \# 1-\mathrm{Nd}(1)-\mathrm{O}(1 \mathrm{~B})$ & $71.0(5)$ & $\mathrm{O}(1 \mathrm{C})-\mathrm{Nd}(1)-\mathrm{O}(1 \mathrm{~B})$ & $86.6(6)$ \\
$\mathrm{O}(2 \mathrm{~B})-\mathrm{Nd}(1)-\mathrm{O}(1)$ & $85.7(6)$ & $\mathrm{O}(2 E) \# 1-\mathrm{Nd}(1)-\mathrm{O}(1)$ & $72.1(6)$ \\
$\mathrm{O}(1 \mathrm{C})-\mathrm{Nd}(1)-\mathrm{O}(1)$ & $143.1(5)$ & $\mathrm{O}(1 \mathrm{~B})-\mathrm{Nd}(1)-\mathrm{O}(1)$ & $106.9(6)$ \\
$\mathrm{O}(2 \mathrm{~B})-\mathrm{Nd}(1)-\mathrm{N}(1 \mathrm{~B})$ & $133.7(6)$ & $\mathrm{O}(2 E) \# 1-\mathrm{Nd}(1)-\mathrm{N}(1 \mathrm{~B})$ & $118.0(6)$ \\
$\mathrm{O}(1 \mathrm{C})-\mathrm{Nd}(1)-\mathrm{N}(1 \mathrm{~B})$ & $136.0(5)$ & $\mathrm{O}(1 \mathrm{~B})-\mathrm{Nd}(1)-\mathrm{N}(1 \mathrm{~B})$ & $66.1(6)$ \\
$\mathrm{O}(1)-\mathrm{Nd}(1)-\mathrm{N}(1 \mathrm{~B})$ & $79.9(5)$ & $\mathrm{O}(2 \mathrm{~B})-\mathrm{Nd}(1)-\mathrm{N}(2 \mathrm{~B})$ & $67.5(6)$ \\
$\mathrm{O}(2 E) \# 1-\mathrm{Nd}(1)-\mathrm{N}(2 \mathrm{~B})$ & $142.1(6)$ & $\mathrm{O}(1 \mathrm{C})-\mathrm{Nd}(1)-\mathrm{N}(2 \mathrm{~B})$ & $124.2(5)$ \\
$\mathrm{O}(1 \mathrm{~B})-\mathrm{Nd}(1)-\mathrm{N}(2 \mathrm{~B})$ & $131.7(5)$ & $\mathrm{O}(1)-\mathrm{Nd}(1)-\mathrm{N}(2 \mathrm{~B})$ & $71.9(5)$ \\
$\mathrm{N}(1 \mathrm{~B})-\mathrm{Nd}(1)-\mathrm{N}(2 \mathrm{~B})$ & $66.3(6)$ & & \\
\end{tabular}

by a single $\mathrm{OAc}^{-}$unit is $6.467 \AA$. A view of the resulting 1-D framework structure is shown in Fig. 4. The average distances for $\mathrm{Yb}-\mathrm{O}$ (phenolic) (2.292 $\AA$ ) and $\mathrm{Yb}-\mathrm{N}(2.533 \AA)$ bonds are comparable to those found in the literature. ${ }^{6,12}$ In 4 , the salen ligand displays two bonding modes with $\mathrm{Yb}^{3+}$ ions: (a) type I Scheme 2, and (b) coordinating with two $\mathrm{Yb}^{3+}$ ions by the $\mathrm{N}_{2} \mathrm{O}_{2}$ set to one $\mathrm{Yb}^{3+}$ and to the other via a phenolic oxygen atom, respectively (Scheme 2, type III).

$\mathbf{T b}_{3}$. Reaction of the methoxy substituted salen derivative $\mathrm{H}_{2} \mathrm{~L}^{\prime}$ with $\mathrm{Tb}(\mathrm{OAc})_{3} \cdot 4 \mathrm{H}_{2} \mathrm{O}$ in the presence of $\mathrm{Et}_{3} \mathrm{~N}$ in refluxing $\mathrm{MeOH}$ produces the 1-D polymeric compound $\left\{\left[\mathrm{Tb}_{3}\left(\mathrm{~L}^{\prime}\right)_{2}(\mathrm{OAc})_{5}\right] \cdot \mathrm{Et}_{2} \mathrm{O} \cdot(\mathrm{MeOH})_{0.5}\right\}_{\mathrm{n}}$ (5). A view of the central 
Table 4 Selected bond lengths $(\AA)$ and angles $\left({ }^{\circ}\right)$ for 3. Symmetry operator $(1+x, y, 1+z)$ generates equivalent atoms marked with "\#”

\begin{tabular}{llll}
\hline $\operatorname{Er}(1)-\mathrm{O}(2 \mathrm{~B})$ & $2.263(7)$ & $\operatorname{Er}(1)-\mathrm{O}(2 E) \# 1$ & $2.334(7)$ \\
$\operatorname{Er}(1)-\mathrm{O}(1 \mathrm{C})$ & $2.345(6)$ & $\operatorname{Er}(1)-\mathrm{O}(1)$ & $2.356(7)$ \\
$\operatorname{Er}(1)-\mathrm{O}(1 \mathrm{~B})$ & $2.353(7)$ & $\operatorname{Er}(1)-\mathrm{O}(3)$ & $2.383(5)$ \\
$\operatorname{Er}(1)-\mathrm{N}(1 \mathrm{~B})$ & $2.493(9)$ & $\operatorname{Er}(1)-\mathrm{N}(2 \mathrm{~B})$ & $2.597(8)$ \\
$\operatorname{Er}(2)-\mathrm{O}(2 \mathrm{D})$ & $2.262(7)$ & $\operatorname{Er}(2)-\mathrm{O}(1 E)$ & $2.253(6)$ \\
$\operatorname{Er}(2)-\mathrm{O}(1 \mathrm{D})$ & $2.307(7)$ & $\operatorname{Er}(2)-\mathrm{O}(2 \mathrm{C})$ & $2.321(7)$ \\
$\operatorname{Er}(2)-\mathrm{O}(2 \mathrm{~F})$ & $2.386(8)$ & $\mathrm{Er}(2)-\mathrm{N}(2 \mathrm{D})$ & $2.483(9)$ \\
$\operatorname{Er}(2)-\mathrm{O}(9)$ & $2.513(7)$ & $\operatorname{Er}(2)-\mathrm{N}(1 \mathrm{D})$ & $2.611(9)$ \\
$\mathrm{O}(2 \mathrm{~B})-\mathrm{Er}(1)-\mathrm{O}(2 E) \# 1$ & $96.2(2)$ & $\mathrm{O}(2 \mathrm{~B})-\operatorname{Er}(1)-\mathrm{O}(1 \mathrm{C})$ & $74.1(2)$ \\
$\mathrm{O}(2 E) \# 1-\operatorname{Er}(1)-\mathrm{O}(1 \mathrm{C})$ & $79.5(2)$ & $\mathrm{O}(2 \mathrm{~B})-\operatorname{Er}(1)-\mathrm{O}(1)$ & $83.0(3)$ \\
$\mathrm{O}(2 E) \# 1-\operatorname{Er}(1)-\mathrm{O}(1)$ & $73.7(2)$ & $\mathrm{O}(1 \mathrm{C})-\operatorname{Er}(1)-\mathrm{O}(1)$ & $142.4(2)$ \\
$\mathrm{O}(2 \mathrm{~B})-\operatorname{Er}(1)-\mathrm{O}(1 \mathrm{~B})$ & $153.3(2)$ & $\mathrm{O}(2 E) \# 1-\operatorname{Er}(1)-\mathrm{O}(1 \mathrm{~B})$ & $69.0(2)$ \\
$\mathrm{O}(1 \mathrm{C})-\operatorname{Er}(1)-\mathrm{O}(1 \mathrm{~B})$ & $81.1(2)$ & $\mathrm{O}(1)-\operatorname{Er}(1)-\mathrm{O}(1 \mathrm{~B})$ & $112.1(2)$ \\
$\mathrm{O}(2 \mathrm{~B})-\operatorname{Er}(1)-\mathrm{N}(1 \mathrm{~B})$ & $135.9(3)$ & $\mathrm{O}(2 E) \# 1-\operatorname{Er}(1)-\mathrm{N}(1 \mathrm{~B})$ & $116.8(3)$ \\
$\mathrm{O}(1 \mathrm{C})-\operatorname{Er}(1)-\mathrm{N}(1 \mathrm{~B})$ & $137.0(3)$ & $\mathrm{O}(1)-\operatorname{Er}(1)-\mathrm{N}(1 \mathrm{~B})$ & $79.5(3)$ \\
$\mathrm{O}(1 \mathrm{~B})-\operatorname{Er}(1)-\mathrm{N}(1 \mathrm{~B})$ & $70.3(3)$ & $\mathrm{O}(2 \mathrm{~B})-\operatorname{Er}(1)-\mathrm{N}(2 \mathrm{~B})$ & $69.1(3)$ \\
$\mathrm{O}(2 E) \# 1-\operatorname{Er}(1)-\mathrm{N}(2 \mathrm{~B})$ & $143.1(3)$ & $\mathrm{O}(1 \mathrm{C})-\operatorname{Er}(1)-\mathrm{N}(2 \mathrm{~B})$ & $124.7(2)$ \\
$\mathrm{O}(1)-\operatorname{Er}(1)-\mathrm{N}(2 \mathrm{~B})$ & $71.1(3)$ & $\mathrm{O}(1 \mathrm{~B})-\operatorname{Er}(1)-\mathrm{N}(2 \mathrm{~B})$ & $135.7(3)$ \\
$\mathrm{N}(1 \mathrm{~B})-\operatorname{Er}(1)-\mathrm{N}(2 \mathrm{~B})$ & $66.9(3)$ & & \\
\hline
\end{tabular}

Table 5 Selected bond lengths ( $\AA$ ) and angles for $\mathbf{4}$. Symmetry operator $(0.5+x, y, z)$ generates equivalent atoms marked with "\#"

\begin{tabular}{llll}
\hline $\mathrm{Yb}(1)-\mathrm{O}(1)$ & $2.207(5)$ & $\mathrm{Yb}(1)-\mathrm{O}(3)$ & $2.299(5)$ \\
$\mathrm{Yb}(1)-\mathrm{O}(2)$ & $2.341(5)$ & $\mathrm{Yb}(1)-\mathrm{O}(7)$ & $2.434(5)$ \\
$\mathrm{Yb}(1)-\mathrm{O}(5)$ & $2.446(5)$ & $\mathrm{Yb}(1)-\mathrm{O}(8)$ & $2.496(5)$ \\
$\mathrm{Yb}(1)-\mathrm{N}(1)$ & $2.543(6)$ & $\mathrm{Yb}(1)-\mathrm{N}(2)$ & $2.551(6)$ \\
$\mathrm{Yb}(2)-\mathrm{O}(4) \# 1$ & $2.244(5)$ & $\mathrm{Yb}(2)-\mathrm{O}(1 \mathrm{~B})$ & $2.281(5)$ \\
$\mathrm{Yb}(2)-\mathrm{O}(2 \mathrm{~B})$ & $2.339(5)$ & $\mathrm{Yb}(2)-\mathrm{O}(5)$ & $2.432(5)$ \\
$\mathrm{Yb}(2)-\mathrm{O}(2)$ & $2.460(5)$ & $\mathrm{Yb}(2)-\mathrm{O}(6)$ & $2.489(5)$ \\
$\mathrm{Yb}(2)-\mathrm{N}(2 \mathrm{~B})$ & $2.497(6)$ & $\mathrm{Yb}(2)-\mathrm{N}(1 \mathrm{~B})$ & $2.540(6)$ \\
$\mathrm{O}(1)-\mathrm{Yb}(1)-\mathrm{O}(2)$ & $148.90(17)$ & $\mathrm{O}(1)-\mathrm{Yb}(1)-\mathrm{N}(1)$ & $72.02(19)$ \\
$\mathrm{O}(2)-\mathrm{Yb}(1)-\mathrm{N}(1)$ & $138.68(19)$ & $\mathrm{O}(1)-\mathrm{Yb}(1)-\mathrm{N}(2)$ & $138.2(2)$ \\
$\mathrm{O}(2)-\mathrm{Yb}(1)-\mathrm{N}(2)$ & $71.58(19)$ & $\mathrm{N}(1)-\mathrm{Yb}(1)-\mathrm{N}(2)$ & $67.1(2)$ \\
$\mathrm{O}(1 \mathrm{~B})-\mathrm{Yb}(2)-\mathrm{O}(2 \mathrm{~B})$ & $149.71(18)$ & $\mathrm{O}(1 \mathrm{~B})-\mathrm{Yb}(2)-\mathrm{O}(2)$ & $75.11(17)$ \\
$\mathrm{O}(2 \mathrm{~B})-\mathrm{Yb}(2)-\mathrm{O}(2)$ & $77.55(17)$ & $\mathrm{O}(1 \mathrm{~B})-\mathrm{Yb}(2)-\mathrm{N}(2 \mathrm{~B})$ & $139.3(2)$ \\
$\mathrm{O}(2 \mathrm{~B})-\mathrm{Yb}(2)-\mathrm{N}(2 \mathrm{~B})$ & $70.32(19)$ & $\mathrm{O}(2)-\mathrm{Yb}(2)-\mathrm{N}(2 \mathrm{~B})$ & $144.23(18)$ \\
$\mathrm{O}(1 \mathrm{~B})-\mathrm{Yb}(2)-\mathrm{N}(1 \mathrm{~B})$ & $72.3(2)$ & $\mathrm{O}(2 \mathrm{~B})-\mathrm{Yb}(2)-\mathrm{N}(1 \mathrm{~B})$ & $137.94(19)$ \\
$\mathrm{O}(2)-\mathrm{Yb}(2)-\mathrm{N}(1 \mathrm{~B})$ & $139.91(19)$ & $\mathrm{N}(2 \mathrm{~B})-\mathrm{Yb}(2)-\mathrm{N}(1 \mathrm{~B})$ & $67.9(2)$ \\
\hline
\end{tabular}

Table 6 Selected bond lengths $(\AA)$ and angles $\left({ }^{\circ}\right)$ for 5

\begin{tabular}{llll}
\hline $\mathrm{Tb}(1)-\mathrm{O}(2)$ & $2.227(5)$ & $\mathrm{Tb}(1)-\mathrm{O}(3)$ & $2.376(5)$ \\
$\mathrm{Tb}(1)-\mathrm{N}(1)$ & $2.506(6)$ & $\mathrm{Tb}(1)-\mathrm{N}(2)$ & $2.518(6)$ \\
$\mathrm{Tb}(2)-\mathrm{O}(3)$ & $2.411(5)$ & $\mathrm{Tb}(2)-\mathrm{O}(6)$ & $2.425(5)$ \\
$\mathrm{Tb}(2)-\mathrm{O}(5)$ & $2.544(5)$ & $\mathrm{Tb}(2)-\mathrm{O}(4)$ & $2.552(5)$ \\
$\mathrm{Tb}(3)-\mathrm{O}(7)$ & $2.232(5)$ & $\mathrm{Tb}(3)-\mathrm{O}(6)$ & $2.395(5)$ \\
$\mathrm{Tb}(3)-\mathrm{N}(3)$ & $2.491(7)$ & $\mathrm{Tb}(3)-\mathrm{N}(4)$ & $2.514(7)$ \\
$\mathrm{O}(2)-\mathrm{Tb}(1)-\mathrm{O}(9)$ & $103.4(2)$ & $\mathrm{O}(2)-\mathrm{Tb}(1)-\mathrm{O}(3)$ & $146.22(18)$ \\
$\mathrm{O}(9)-\mathrm{Tb}(1)-\mathrm{O}(3)$ & $85.89(19)$ & $\mathrm{O}(2)-\mathrm{Tb}(1)-\mathrm{N}(1)$ & $72.9(2)$ \\
$\mathrm{O}(9)-\mathrm{Tb}(1)-\mathrm{N}(1)$ & $83.6(2)$ & $\mathrm{O}(3)-\mathrm{Tb}(1)-\mathrm{N}(1)$ & $140.87(19)$ \\
$\mathrm{O}(2)-\mathrm{Tb}(1)-\mathrm{N}(2)$ & $140.40(19)$ & $\mathrm{O}(3)-\mathrm{Tb}(1)-\mathrm{N}(2)$ & $72.91(18)$ \\
$\mathrm{N}(1)-\mathrm{Tb}(1)-\mathrm{N}(2)$ & $68.1(2)$ & $\mathrm{O}(3)-\mathrm{Tb}(2)-\mathrm{O}(6)$ & $146.90(17)$ \\
$\mathrm{O}(14)-\mathrm{Tb}(2)-\mathrm{O}(5)$ & $130.95(17)$ & $\mathrm{O}(17)-\mathrm{Tb}(2)-\mathrm{O}(5)$ & $129.20(16)$ \\
$\mathrm{O}(3)-\mathrm{Tb}(2)-\mathrm{O}(5)$ & $89.56(17)$ & $\mathrm{O}(6)-\mathrm{Tb}(2)-\mathrm{O}(5)$ & $63.00(17)$ \\
$\mathrm{O}(12)-\mathrm{Tb}(2)-\mathrm{O}(5)$ & $72.65(17)$ & $\mathrm{O}(15)-\mathrm{Tb}(2)-\mathrm{O}(5)$ & $140.05(17)$ \\
$\mathrm{O}(14)-\mathrm{Tb}(2)-\mathrm{O}(4)$ & $129.98(16)$ & $\mathrm{O}(17)-\mathrm{Tb}(2)-\mathrm{O}(4)$ & $134.70(16)$ \\
$\mathrm{O}(3)-\mathrm{Tb}(2)-\mathrm{O}(4)$ & $62.69(15)$ & $\mathrm{O}(6)-\mathrm{Tb}(2)-\mathrm{O}(4)$ & $87.65(16)$ \\
$\mathrm{O}(12)-\mathrm{Tb}(2)-\mathrm{O}(4)$ & $137.09(17)$ & $\mathrm{O}(15)-\mathrm{Tb}(2)-\mathrm{O}(4)$ & $75.60(16)$ \\
$\mathrm{O}(5)-\mathrm{Tb}(2)-\mathrm{O}(4)$ & $64.49(16)$ & & \\
\hline
\end{tabular}

$\mathrm{Tb}_{3}$ core of $\mathbf{5}$ is shown in Fig. 5. The central $\mathrm{Tb}_{3}$ core of this material contains two $\mathrm{L}^{\prime 2-}$ groups bound to the two terminal $\mathrm{Tb}^{3+}$ ions via the conventional $\mathrm{N}_{2} \mathrm{O}_{2}$ binding pocket as well as one chelating acetate and are 8-coordinate. The central $\mathrm{Tb}$ ion $(\mathrm{Tb}(2))$ is coordinated to 10 oxygen atoms. Each $\mathrm{L}^{\prime 2-}$ group binds $\mathrm{Tb}(2)$ with a bridging phenolic $\mathrm{O}$ atom as well as one of its OMe groups.

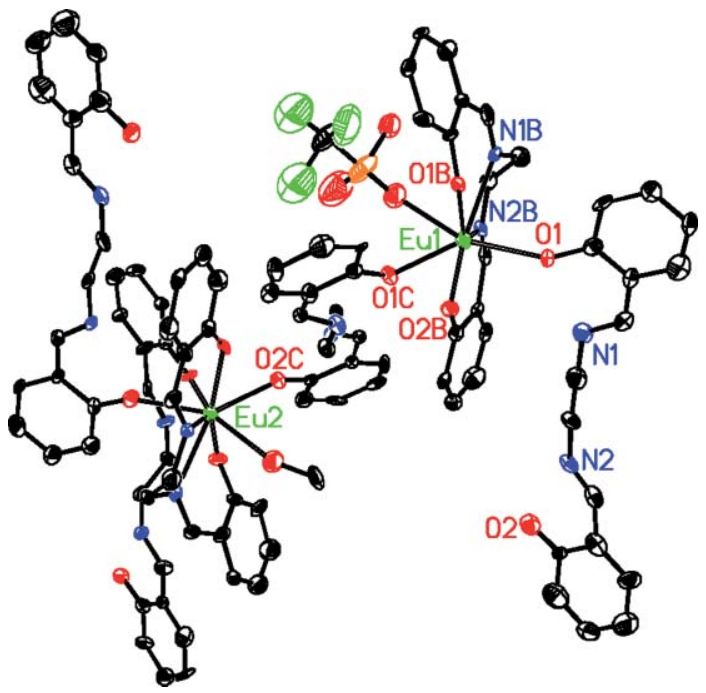

Fig. 1 A view of the molecular structure of $\mathbf{1} . \mathrm{H}$ atoms have been omitted for clarity and thermal ellipsoids drawn at the $50 \%$ probability level.

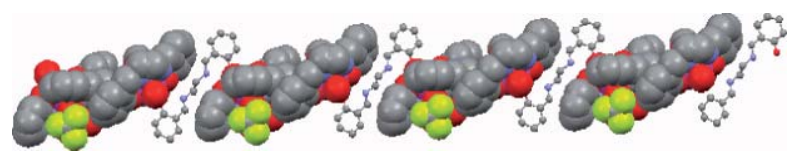

Fig. 2 A view of the 1-D polymeric chain in $\mathbf{1}$.

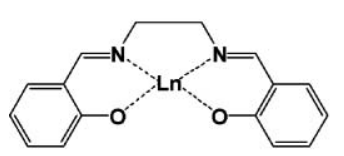<smiles>CCCCCOc1ccccc1/C=N/C/N=C/c1ccccc1O</smiles>

Type-I

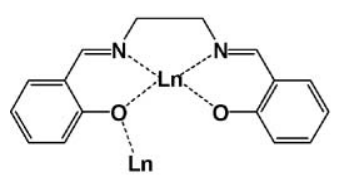

Type-II

Type-III

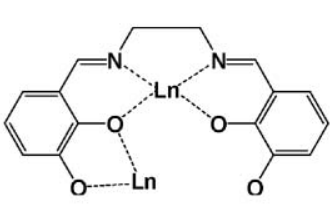

Type-IV

Scheme 2 Four bonding modes for Schiff-base ligands with $\mathrm{Ln}^{3+}$ ions in 1-5.

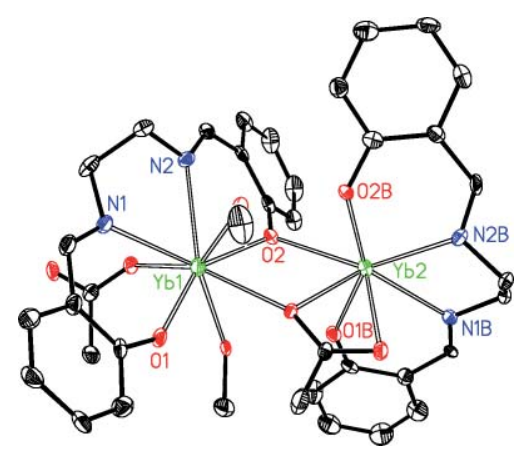

Fig. 3 A view of the molecular structure of 4 . $\mathrm{H}$ atoms have been omitted for clarity and thermal ellipsoids drawn at the $50 \%$ probability level. 


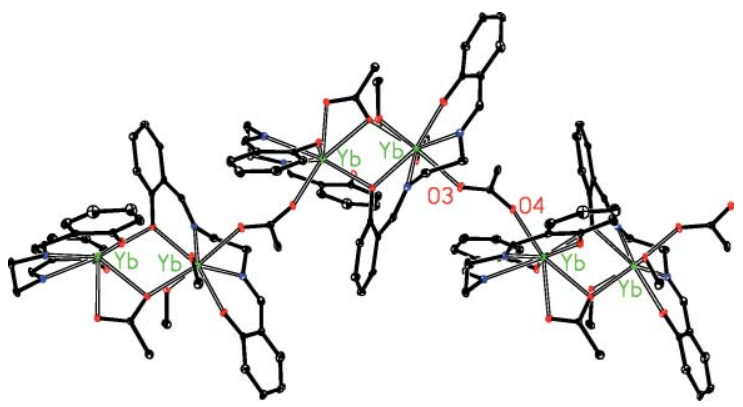

Fig. 4 A view of the 1-D polymeric chain in 4.

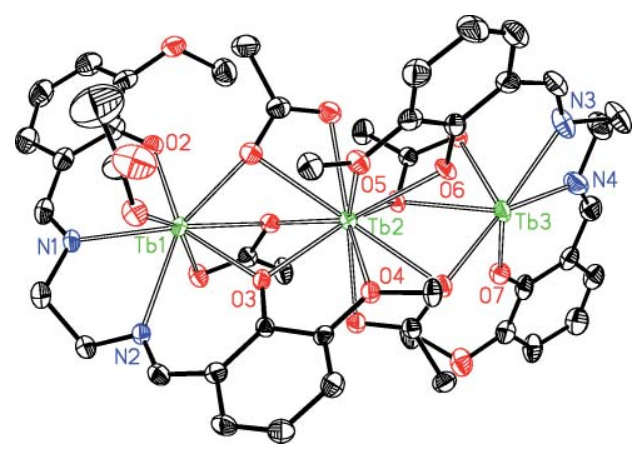

Fig. 5 A view of the molecular structure of 5 . H atoms have been omitted for clarity and thermal ellipsoids drawn at the $50 \%$ probability level.

There are also two chelating $\mathrm{OAc}^{-}$anions each of which bridges to a terminal $\mathrm{Tb}^{3+}$ ion via one $\mathrm{O}$ atom. In turn, each terminal $\mathrm{Tb}^{3+}$ ion bears a chelating $\mathrm{OAc}^{-}$group which also bridges to the central $\mathrm{Tb}$ ion via one $\mathrm{O}$ atom. The 1-D polymeric framework is completed by $\mathrm{OAc}^{-}$units which link the $\mathrm{Tb}_{3}$ clusters. (Fig. 6) $\mathrm{The} \mathrm{Tb}-\mathrm{Tb}$ distances within the central $\mathrm{Tb}_{3}$ cluster are similar at $3.709 \AA$ and $3.719 \AA$ for $\mathrm{Tb}(1)-\mathrm{Tb}(2)$ and $\mathrm{Tb}(2)-\mathrm{Tb}(3)$ respectively. The average distances for $\mathrm{Tb}-\mathrm{O}$ (phenolic) $(2.344 \AA$ ), $\mathrm{Tb}-\mathrm{O}$ (methoxy) (2.549 $\AA$ ) and $\mathrm{Tb}-\mathrm{N}(2.507 \AA)$ are comparable to those found in the literature. ${ }^{6,12}$ As expected, the average $\mathrm{Tb}-\mathrm{O}$ (phenolic) distance is significantly shorter than the average $\mathrm{Tb}-\mathrm{O}$ (methoxy) distance and reflects the difference between the two different oxygen atoms involved. In this case the modified salen ligand $\mathrm{H}_{2} \mathrm{~L}^{\prime}$ bears two extra -OMe groups and has only one overall bonding mode. This involves coordination to two $\mathrm{Tb}^{3+}$ ions by the $\mathrm{N}_{2} \mathrm{O}_{2}$ core plus two $\mathrm{O}$ atoms - one from a phenolic group and one from an OMe unit (Scheme 2, type IV).

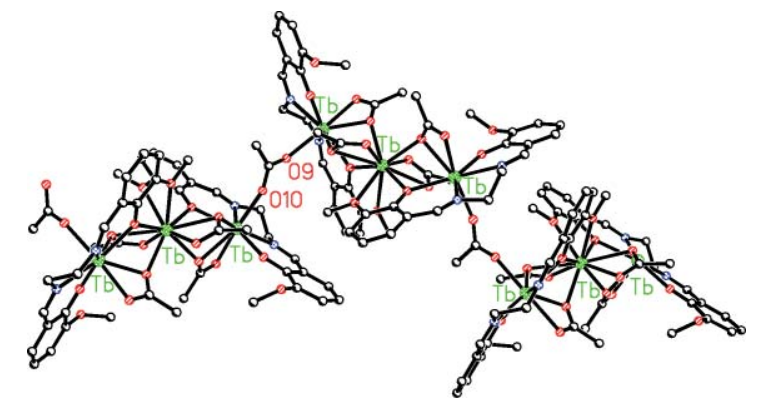

Fig. 6 A view of the 1-D polymeric chain in $\mathbf{5}$.

We have also probed the nature of these compounds in solution using high resolution ESI mass spectrometry studies.
As expected, in solution the 1-D polymeric structures of the complexes break down into smaller, more soluble units. Thus the ESI mass spectra of complexes 1-3, $\mathbf{4}$ and $\mathbf{5}$ show $\left[\mathrm{Ln}_{2} \mathrm{~L}_{2}\left(\mathrm{H}_{2} \mathrm{~L}\right)_{4}\right]^{2+}$, $\left[\mathrm{Yb}_{2} \mathrm{~L}_{2}(\mathrm{OAc})(\mathrm{MeOH})_{2}\right]^{+}$and $\left[\mathrm{Tb}_{3} \mathrm{~L}^{\prime}\left(\mathrm{HL}^{\prime}\right)(\mathrm{OAc})_{5}\right]^{+}$fragments, respectively.

\section{Luminescence Studies}

The photophysical properties of the Eu(III) compound $\mathbf{1}$ and $\mathrm{Tb}$ (III) compound 5 were studied in $\mathrm{MeOH}$. The free $\mathrm{H}_{2} \mathrm{~L}$ and $\mathrm{H}_{2} \mathrm{~L}^{\prime}$ ligands exhibit absorption bands in their uv-vis spectra (i.e. 216,255 and $316 \mathrm{~nm}$ for $\mathrm{H}_{2} \mathrm{~L}$ and 218, 260, 286, 323 and $413 \mathrm{~nm}$ for $\mathrm{H}_{2} \mathrm{~L}^{\prime}$ ). These bands are red-shifted upon metal ion coordination in both 1 and 5 (Fig. 7). The emission and excitation spectra of both $\mathrm{H}_{2} \mathrm{~L}$ and $\mathbf{1}$ are shown in Fig. 8. Excitation of the absorption band at $366 \mathrm{~nm}$ in the free ligand $\mathrm{H}_{2} \mathrm{~L}$ produces a broad emission band at $\lambda_{\max }=455 \mathrm{~nm}$. Excitation of the ligand centered absorption band at $271 \mathrm{~nm}$ in 1 results in visible emission bands for the $\mathrm{Eu}^{3+}$ ion $\left({ }^{5} \mathrm{D}_{0} \rightarrow{ }^{7} \mathrm{~F}_{\mathrm{j}}\right.$ transitions, $\mathrm{j}=1,2,3$ and 4$)$. The fluorescence of 1 is very weak with quantum yield $\left(\Phi_{\mathrm{em}}\right)^{13}$ less than $10^{-3}$. The emission and excitation spectra of both $\mathrm{H}_{2} \mathrm{~L}^{\prime}$ and $\mathbf{5}$ are shown in Fig. 9. Excitation of the absorption band at $361 \mathrm{~nm}$ in the free ligand $\mathrm{H}_{2} \mathrm{~L}^{\prime}$ produces a broad emission band at $\lambda_{\max }=490 \mathrm{~nm}$. Excitation of the ligand centered absorption band at $340 \mathrm{~nm}$ in 5 results in typical visible emission bands for the $\mathrm{Tb}^{3+}$ ion $\left({ }^{5} \mathrm{D}_{4} \rightarrow\right.$ ${ }^{7} \mathrm{~F}_{\mathrm{n}}$ transitions, $n=6,5,4$ and 3 ). The fluorescence quantum yield $\left(\Phi_{\mathrm{em}}\right)^{14}$ for 5 is 0.21 .

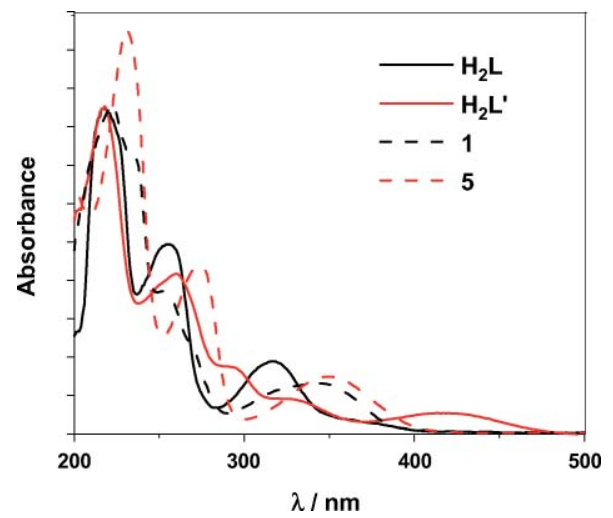

Fig. 7 UV-Vis spectra of free ligands $\left(\mathrm{H}_{2} \mathrm{~L}\right.$ and $\left.\mathrm{H}_{2} \mathrm{~L}^{\prime}\right), \mathbf{1}$ and 5 in $\mathrm{CH}_{3} \mathrm{OH}$. (Concentrations: $\sim 10^{-6} \mathrm{M}$ ).

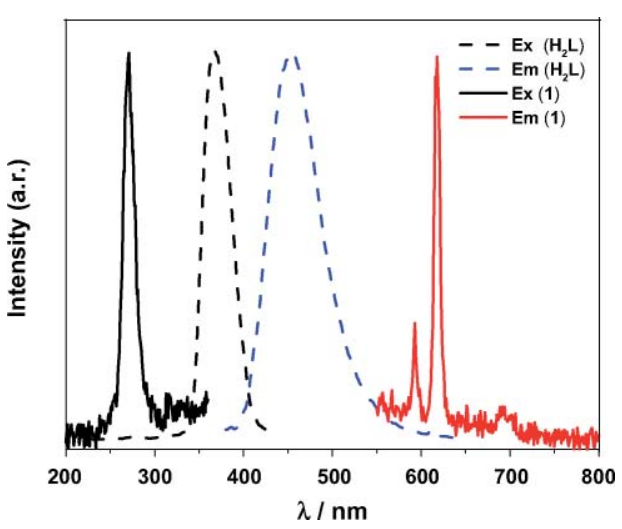

Fig. 8 The excitation and emission spectra of free $\mathrm{H}_{2} \mathrm{~L}(---)$ and $\mathbf{1}(-)$. 


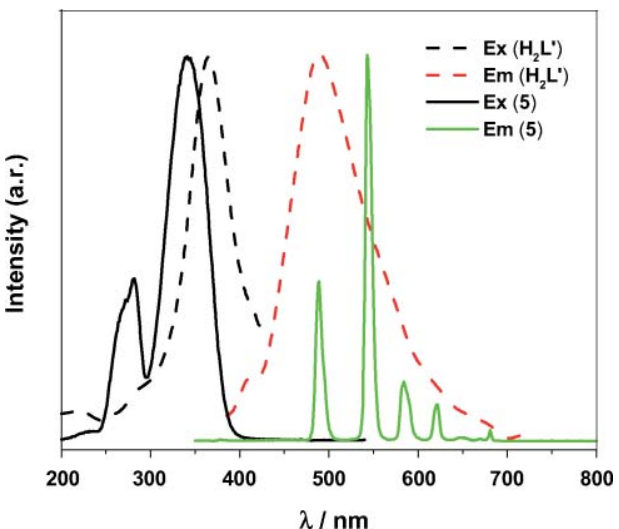

Fig. 9 The excitation and emission spectra of free $\mathrm{H}_{2} \mathrm{~L}^{\prime}(---)$ and $5(-)$.

\section{Conclusions}

Five 1-D coordination lanthanide polymers with Schiff-base ligands $\mathrm{H}_{2} \mathrm{~L}$ and $\mathrm{H}_{2} \mathrm{~L}^{\prime}$ have been prepared, and structurally characterized. The stoichiometry and structures of these compounds are dependent on the Schiff-base ligand employed in their syntheses as well as the nature of the anion present $\left(\mathrm{CF}_{3} \mathrm{SO}_{3}{ }^{-} v s\right.$. OAc $\left.{ }^{-}\right)$. In 1-3, the flexible neutral salen ligand $\left(\mathrm{H}_{2} \mathrm{~L}\right)$ acts as a bridge or linker in the formation of 1-D coordination frameworks, while $\mathrm{CF}_{3} \mathrm{SO}_{3}{ }^{-}$is merely a counter anion. In $\mathbf{4}$ and $\mathbf{5}$, acetate $\left(\mathrm{OAc}^{-}\right)$is employed as a linker in the formation of such frameworks. In $\mathbf{1}$ and $\mathbf{5}$ both sets of ligand frameworks enable the materials to display luminescence of the lanthanide(III) ions via the antenna effect.

\section{Acknowledgements}

We thank Hong Kong Baptist University (FRG/06-07/II-16), the Hong Kong Research Grants Council(HKBU 202407), the Robert A. Welch Foundation (Grant F-816), the Texas Higher Education Coordinating Board (ARP 003658-0010-2006) and the Petroleum Research Fund, administered by the American Chemical Society (47014-AC5) for financial support.

\section{Notes and references}

1 (a) R. J. Hill, D. L. Long, P. Hubberstey, M. Schroder and N. R. Champness, J. Solid State Chem., 2005, 178, 2414; (b) J.-C. G. Bünzli and C. Piguet, Chem. Soc. Rev., 2005, 34, 1048; (c) K. Kuriki, Y. Koike and Y. Okamoto, Chem. Rev., 2002, 102, 2347.

2 (a) J.-C. G. Bünzli, Lanthanides Probes in Life, Chemical and Earth Sciences, Elsevier, Amsterdam, The Netherlands, 1989; Vol. 1989; (b) C. Piguet and J.-C. G. Bünzli, Chem. Soc. Rev., 1999, 28, 347.

3 (a) J. M. Lehn, Supramolecular Chemistry: Concepts and Perspectives, VCH, Weinheim, 1995; (b) C. Piguet, Chimia, 1996, 50, 144; (c) P. Guerriero, S. Tamburini and P. A. Vigato, Coord. Chem. Rev., 1995, 139, 17; (d) Z.-P. Zheng, Chem. Commun., 2001, 2521.
4 (a) N. L. Rosi, J. Kim, M. Eddaoudi, B. L. Chen, M. O'Keeffe and O. M. Yaghi, J. Am. Chem. Soc., 2005, 127, 1504; (b) L. P. Wu, M. Munakata, M. Yamamoto, T. Kuroda-Sowa and M. Maekawa, J. Coord. Chem., 1996, 37, 361; (c) M. Sakamoto, K. Manseki and H. Ōkawa, Coord. Chem. Rev., 2001, 219-221, 379; (d) D. T. de Lill, A. de Bettencourt-Dias and C. L. Cahill, Inorg. Chem., 2007, 46, 3960; (e) R. Shunmugam and G. N. Tew, J. Am. Chem. Soc., 2005, 127(39), 13567; (f) T. M. Reineke, M. Eddaoudi, M. Fehr, D. Kelley and O. M. Yaghi, J. Am. Chem. Soc. 1999, 121, 1651; (g) A. Deluzet, W. Maudez, C. Daiguebonne and O. Guillou, Cryst. Growth Des., 2003, 3, 475; (h) L. Pan, M. Zheng, Y. Wu, S. Han, R. Yang, X. Huang and J. Li, Inorg. Chem., 2001, 40, 828; (i) C. Serre, F. Millange, J. Marrot and G. Férey, Chem. Mater., 2002, 14, 2409; (j) X. Guo, G. Zhu, F. Sun, Z. Li, X. Zhao, X. Li, H. Wang and S. Qiu, Inorg. Chem., 2006, 45, 2581; (k) R. Cao, D. Sun, Y. Liang, M. Hong, K. Tatsumi and Q. Shi, Inorg. Chem., 2002, 41, 2087; (l) W. Lin, Z. Wang and L. Ma, J. Am. Chem. Soc., 1999, 121, 11249; $(m)$ O. R. Evans, R. Xiong, Z. Wang, G. K. Wong and W. Lin, Angew. Chem., Int. Ed., 1999, 38, 536.

5 (a) A. Y. Robin and K. M. Fromm, Coord. Chem. Rev., 2006, 250, 2127; (b) Y.-B. Xie, C. Zhang, J.-R. Li and X.-H. Bu, Dalton Trans., 2004, 562; (c) Y. Zheng, M. Du, J.-R. Li, R.-H. Zhang and X.-H. Bu, Dalton Trans., 2003, 1509; (d) X.-H. Bu, W. Chen, W.-F. Hou, M. Du, R.-H. Zhang and F. Brisse, Inorg. Chem, 2002, 41, 3477; (e) X.-H. Bu, W.-F. Hou, M. Du, W. Chen and R.-H. Zhang, Cryst. Growth Des, 2002, 2, 303; (f) L. Carlucci, G. Ciani, D. M. Proserpio and S. Rizzato, CrystEngComm, 2002, 4, 413; (g) G. A. Van Albada, G. A. Guijt, R. C. Haasnoot, J. G. Lutz, M. Spek and J. Reedijk, Eur. J. Inorg. Chem., 2000, 121; (h) L. G. Carlucci, D. W. Ciani, V. Gudenberg and D. Proserpio, Inorg. Chem., 1997, 36, 3812; (i) J. R. Blake, N. R. Champness, W. Levason and G. Reid, Inorg. Chem., 1996, 35, 4432; (j) J. R. Blake, N. R. Champness, W. Levason and G. Reid, Chem. Commun., 1995, 1277; (k) J.-R. Li, X.-H. Bu and R.-H. Zhang, Inorg. Chem., 2004, 43, 237; (l) D. M. L. Goodgame, S. Menzer, A. M. Smith and D. J. Williams, Chem. Commun., 1997, 339; (m) P.-R. Wei, D.-D. Wu, Z.-Y. Zhou and T. C. W. Mak, Polyhedron, 1998, 17, 497.

6 (a) X.-P. Yang, R. A. Jones and W.-K. Wong, Chem. Commun., 2008, 3266; (b) X.-P. Yang, B. P. Hahn, R. A. Jones, W.-K. Wong and K. J. Stevenson, Inorg. Chem., 2007, 46, 7050; (c) X.-P. Yang, R. A. Jones, W.-K. Wong, V. Lynch, M. M. Oye and A. L. Holmes, Chem. Commun., 2006, 1836; (d) W.-K. Wong, X.-P. Yang, R. A. Jones, J. H. Rivers, V. Lynch, W.-K. Lo, D. Xiao, M. M. Oye and A. L. Holmes, Inorg. Chem., 2006, 45, 4340; (e) X.-P. Yang, B. P. Hahn, R. A. Jones, K. J. Stevenson, J. S. Swinnea and Q. Wu, Chem. Commun., 2006, 3827; $(f)$ X.-P. Yang and R. A. Jones, J. Am. Chem. Soc., 2005, 127, 7686; (g) X.-P. Yang, R. A. Jones, V. Lynch, M. M. Oye and L. H. Archie, Dalton Trans., $2005,849$.

7 W. Xie, M. J. Heeg and P. G. Wang, Inorg. Chem., 1999, 38, 2541

8 (a) M. P. Hogerheide, J. Boersma and G. V. Konten, Coord. Chem. Rev., 1996, 155, 87; (b) V. Alexander, Chem. Rev., 1995, 95, 273; (c) D. E. Featon and P. A. Vigato, Chem. Soc. Rev., 1988, 17, 69.

9 F. Lam, J.-X. Xu and K. S. Chan, J. Org. Chem., 1996, 61, 8414.

10 G. M. Sheldrick, SHELX, 97, A software package for the solution and refinement of X-ray data, University of Göttingen, Göttingen, Germany, 1997.

11 D. T. Cromer and J. T. Waber, International Tables for X-Ray Crystallography, Kynoch Press, Birmingham, vol. 4, 1974, Table 2.2A.

12 (a) M. Sakamoto, K. Manseki and H. Okawa, Coord. Chem. Rev., 2001, 219-221, 379; (b) J.-P. Cortes, F. Dahan and A. Dupuis, Inorg. Chem., $2000,39,165$.

13 Fluorescence quantum yields were determined relative to $\left[\mathrm{Ru}(\text { bipy })_{3}\right] \mathrm{Cl}_{2}$ in water (bipy $=2,2^{\prime}$-bipyridine; $\Phi_{\mathrm{em}}=0.028$ ): $\mathrm{K}$. Nakamaru, Bull. Chem. Soc. Jpn., 1982, 55, 2697.

14 Fluorescence quantum yields were determined by using quinine sulfate $\left(\Phi_{\mathrm{em}}=0.546 \text { in } 0.5 \mathrm{M} \mathrm{H}_{2} \mathrm{SO}_{4}\right)^{12}$ as standard for the $\mathrm{Tb}^{3+}$ complex: S. R. Meech and D. J. Philips, J. Photochem., 1983, 23, 193. 